\title{
COMITÊ DE ESTUDOS DE MORTE MATERNA: UMA EXPERIÊNCIA DE TRABALHO MULTIPROFESSIONAL.*
}

\author{
Brasileira Cordeiro Lopes** \\ Vânia Muniz Nequer Soares**
}

RESUMO: Os Comitês de Morte Matema fazem parte da proposta de implantação de um Sistema de Vigilância Epidemiológica das complicações da gravidez, destinado a proporcionar informação útil e oportuna, favorecendo desta maneira o uso da mesma a nivel do sistema local de saúde, e conseqüentemente, o fortalecimento da sua capacidade de análise da situação da saúde materna, para adequar sua produção de serviços às necessidades específicas da população. No Paraná, a partir de 1989 foram implantados 24 Comitês Regionais e 1 Comitê Estadual de Morte Materna, os quais têm caráter interinstitucional e multiprofissional. A enfermagem está representada em todos os Comitês, realizando auditoria, avaliação e propostas de ação, para a redução das mortes maternas no Estado do Paraná.

\begin{abstract}
The Maternal Mortality Committees are part of the proposed Epidemiological Vigilance for Pregnancy Complication System implementation, which intends to provide useful and timely information, thus favoring the use of such information on the local health system level, and therefore strengthening its capacity of analyzing the maternal health situation, in order to adjust its service offer to the specific needs of the population. In Paraná, from 1989 on, 24 Regional Committees and 1 State Committee against Maternal Mortality were implemented, involving different institutions and professionals. Nursing is represented in all Committees, performing audits and appraisals and proposing actions which will reduce Maternal Mortality in the State of Paraná.
\end{abstract}

\section{INTRODUÇÃO}

A morte de mulheres em idade fértil por causas ligadas à gravidez, parto e puerpério, atualmente é em sua grande maioria prevenível e evitável.

No entanto, a OMS estima que aproximadamente 500.000 mulheres morram anualmente por complicações ligadas ao ciclo gravídico-puerperal, e que destas, $\mathbf{9 8 \%}$ ocorram nos países em desenvolvimento.

Isto equivale à queda de um avião a cada quatro horas com 250 mulheres a bordo, tendo algumas menos de 20 anos. Todas elas grávidas ou que tenham dado à luz recentemente, sendo que a maioria deixa filhos, órgãos e familiares que dependiam dela. (4)

Nos países em desenvolvimento a morte materna se apresenta como um grave problema social e de saúde.

Morrem na América Latina anualmente cerca de 30.000 gestantes, mas se todas essas mulheres tivessem condições de vida e atenção à saúde semelhantes à dos países desenvolvidos teriam sido evitadas $90 \mathrm{a}$ $95 \%$ destas mortes. (5) (6)

Atualmente, nesta última década do século XX, a mortalidade materna nos países europeus, nos Estados Unidos, Japão, Nova Zelândia e outros de igual nível de vida e de saúde, excepcionalmente atinge 10 por 100.000 nascidos vivos (N.V.). Na Ásia e América Latina são observados valores de até 200 a 400 por 100.000 N.V. - (Tabela 1). ${ }^{(3)}$

No Brasil, em 1987 a taxa oficial foi de 70 óbitos

* Trabalho apresentado como tema livre no $44^{\circ}$ Congresso Brasileiro de Enfermagem. Brasilia, D.F, 1992.

** Enf ermeiras do Instituto de Saúde do Paraná - Membros dos Comitès de Morte Materna do Paraná. 
por 100.000 N.V. Mas as estatísticas brasileiras não refletem a realidade, uma vez que muitos óbitos maternos deixam de ser notificados pelos médicos por ocasião do preenchimento da declaração de óbito, o que leva a uma subnumeração dos óbitos ocorridos. (Tabela 1)

Estima-se que no Brasil morram na realidade cerca de 3.000 mulheres em estado gestacional todos os anos, o que equivale a um coeficiente de 140 por 100.000 N.V.

Em um mesmo país observam-se variações regionais e locais destes coeficientes dependendo das características sócio-econômicas de grupos populacionais. Sabe-se que no Brasil há locais com coeficientes maiores que a média nacional e que não raro atingem 100 por 100.000 N.V. (Tabela 1).

Tabela 1 - Coeficiente de Mortalidade Matema por 100.000 Nascidos Vivos em Alguns Estados Brasileiros e Outros Países. Anos Próximos a 1987.

\begin{tabular}{l|c}
\hline \multicolumn{1}{c|}{ LOCAL } & $\begin{array}{c}\text { Coef. de } \\
\text { Mortalidade Materna } \\
\text { Por 100.000 N.V. }\end{array}$ \\
\hline Paraná & 80 \\
\hline Outros Estados & 80 \\
\hline Goiás & 230 \\
Pará & 40 \\
Santa Catarina & 50 \\
São Paulo & 50 \\
Rio Grande do Sul & - \\
Pernambuco & 70 \\
Brasil & \\
\hline Outros Países & 480 \\
\hline Bolivia & 303 \\
Peru & 82 \\
México & 69 \\
Argentina & 48 \\
Chile & 20 \\
Japão & 10 \\
U.S.A. & 4 \\
Suécia & \\
\hline
\end{tabular}

Fonte: Instituto de Saúde do Paraná

Nota: Baseado nas Estatísticas de Registro Civil - ANUÁRIO DO IBGE 1987, WORLD HEALTH STATISTICS - 1988 e Plano Regional para a Redução da Mortalidade Matema nas Américas/ OPAS -Dados Oficiais. ${ }^{(7)}$
O Estado de São Paulo, segundo LAURENTI, apresentou no triênio 1988/90 coeficiente médio de 47,2 por 100.000 N.V., com uma redução de $67,2 \%$ da mortalidade materna em relação ao triênio $1960 / 62$, cujo coeficiente era de 143,9 por 100.000 N.V. ${ }^{(3)}$

No Paraná, os dados oficiais demonstram uma redução gradativa dos óbitos maternos no estado, passando de um coeficiente de 111,02 por 100.000 N.V. em 1979 para 72,93 em 1988. A partir de 1989 no entanto, voltou a aumentar, através da correção das estatísticas oficiais e a inclusão dos óbitos maternos identificados pelo "Comitês Regionais de morte Materna do Paraná", os quais realizam a investigação dos óbitos de mulheres em idade fértil nas suas áreas de abrangência, buscando identificar a sub-notificação de óbitos maternos nas declarações de óbito.

O coeficiente de mortalidade materna passou assim para 100,00 por 100.000 N.V. em 1990 no Paraná, e em 1991 os dados preliminares do Comitê Estadual de Morte Materna mostraram um coeficiente de 95,79 por 100.000 N.V. (Tabela 2)

Tabela 2 - Números de Nascidos Vivos, Óbitos Maternos e Coeficiente de Mortalidade Materna (por 100.000 N.V.) - Paraná - Brasil - 1979-1991.

\begin{tabular}{c|c|c|c}
\hline ANO & $\begin{array}{c}\mathrm{N}^{\circ} \mathrm{DE} \\
\text { NASCIDOS } \\
\text { VIVOS }\end{array}$ & $\begin{array}{c}\mathrm{N}^{\circ} \mathrm{DE} \\
\text { ÓBITOS } \\
\text { MATERNOS }\end{array}$ & $\begin{array}{l}\text { COEFICI. DE } \\
\text { MORTALID. } \\
100.000 \mathrm{~N} . \mathrm{V} .\end{array}$ \\
\hline 1979 & 203.564 & 226 & 111,02 \\
\hline 1980 & 199.755 & 209 & 104,63 \\
\hline 1981 & 204.625 & 192 & 93,83 \\
\hline 1982 & 215.485 & 181 & 83,99 \\
\hline 1983 & 191.065 & 182 & 95,25 \\
\hline 1984 & 174.704 & 184 & 105,32 \\
\hline 1985 & 182.106 & 164 & 90,06 \\
\hline 1986 & 188.033 & 148 & 78,71 \\
\hline 1987 & 179.733 & 150 & 83,46 \\
\hline 1988 & 190.610 & 131 & 72,93 \\
\hline 1989 & 179.623 & 139 & 77,38 \\
\hline 1990 & $179.568^{\star}$ & 180 & 100,24 \\
\hline 1991 & $179.551^{\star}$ & $172^{\star \star}$ & $95,79^{\star}$ \\
\hline
\end{tabular}

Fonte: Instituto de Saúde do Paraná/Sistema Único de Saúde-PR

\footnotetext{
* Dados estimados pelo Comitê Estadual de Morte Matema

** Dados Preliminares levantados pelos Comitês de Morte Materna.
} 


\section{REVISÃO DA LITERATURA}

Segundo a Classificação Internacional de Doenças-CID ( $9^{\mathrm{a}}$ Revisão - 1975), Morte Matema é “a morte de uma mulher durante a gestação ou dentro de um período de 42 dias após o término da gestação, independentemente da duração ou localização da gravidez, devido a qualquer causa relacionada ou agravada pela gravidez, ou por medidas tomadas em relação a ela, porém não devida a causas acidentais ou incidentais".

As taxas de mortalidade materna vêm apresentando um declínio em vários países do mundo, embora continuem sendo muito altas na maioria dos países em desenvolvimento, entre eles os países da América Latina.

Já nas primeiras décadas do século XX, os países do "primeiro mundo", apresentavam indícios da melhoria geral da qualidade de vida e saúde de suas populações, o que logo se refletiu no declínio da mortalidade materna, principalmente a partir da metade do século (década de 40 e 50) quando o uso de antibióticos fez reduzir bastante as complicações infecciosas do puerpério e do aborto. (1) (3)

A idéia de se criar Comitês de Morte Materna não é nova. Já em 1930, na Filadélfia, Dr Willians criava comitês de estudos de morte materna, pois ele acreditava que, a única forma de reduzir estas mortes, era estudando uma por uma e propondo ações para evitálas. (8)

Após estudo cuidadoso de cada óbito, ele procuravadetectar de quem era a responsabilidade e o grau de evitabilidade da morte. Posteriormente, em 1932, Nova Iorque implantava seu comitê, e sua influência se espalhou rapidamente pelos Estados Unidos, $\mathrm{Ca}$ nadá e após a guerra, para vários países da Europa.

$\mathrm{Na}$ Inglaterra, em 1952, iniciou-se a primeira "Investigação Confidencial sobre Mortes Maternas da Inglaterra e País de Gales", cujo relatório foi publicado anos depois. A experiência mais conhecida do trabalho dos Comitês de Morte Materna passou a ser a do Reino Unido, pela qualidade dos dados coletados e pela eficácia das medidas propostas para reduzir as mortes. Estas investigações continuam até hoje, com publicações periódicas de relatórios. (1)

A implantação dos Comitês de Estudos da Mortalidade Materna, atualmente tem sido recomendada a nível internacional, por ser considerado um valioso instrumento do tipo médico-administrativo para detecção e análise dos óbitos maternos e de intervenção na redução dos óbitos por complicações da gravidez, parto ou puerpério.

Os Comitês de Morte materna fazem parte da proposta de implantação de um sistema de vigilância epidemiológica sistemático e contínuo destas mortes, preconizado pela OMS. ${ }^{(6)}$

$\mathrm{Na}$ América Latina, existem várias iniciativas isoladas de implementação e organização de Comitês de Morte Materna. Em fins de 1991, oito países da América Latina e do Caribe, tinham desenvolvido atividades de distinto grau de avanço no sentido de implementação e funcionamento dos Comitês de Mortalidade Materna e/ou Sistema de Vigilância das Mortes Maternas. Destes oito países somente em Cuba existe um "Sistema Nacional de Informação Sobre Mortalidade Materna"; nos outros sete a situação é desigual; assim, no Brasil, nessa época estavam instalados comitês em 04 estados (em São Paulo em 04 universidades, na Bahia em 02 municípios, em Pernambuco em 02 municípios, e em 24 Regionais de Saúde no Estado do Paraná); Costa Rica e Chile tem Comitês Nacionais de Mortalidade Materna, com seus respectivos comitês locais nas principais instituições de saúde e nos hospitais; na Colômbia se instituíram comitês em doze estados e em várias cidades. O México formou comitês em todos os hospitais da Seguridade Social e da Secretaria de Saúde. A Nicarágua tem um sistema de notificação dos óbitos maternos no Sistema Único de Saúde, e o Paraguai também tem um Comitê Nacional.

O Brasil iniciou em 1989 o trabalho de incentivo para criação dos Comitês Estaduais de Morte Materna, através de um grupo de assessores do Ministério da Saúde, que realizaram diversos seminários macroregionais sobre intervenções para redução de Mortalidade Materna. Em maio de 1992, oficializou-se no Brasil o Comitê Nacional de Mortalidade Materna.

O Paraná é o único estado brasileiro que implantou comitês em todo o Estado, contando atualmente com 24 comitês regionais e um Comitê Estadual de Morte Materna, todos organizados e atuantes. Já existe a proposta de uma gradativa implantação de Comitês Municipais e de Comitês Hospitalares de Morte Materna. (1)

\section{IMPLANTAÇÃO DOS COMITÊS DE MORTE MATERNA NO PARANÁ}

A Comissão Interinstitucional de Saúde da Mulher e da Criança do Paraná propôs a implantação dos comitês no Esiado em meados de 1988. (1) 
Em janciro de 1989, com patrocínio da DINSAMI/MS, foi realizado um Simpósio Estadual de Mortc Materna, para o qual foram convidados dois profissionais de cada cidade-sede de macro-regional de saúde, sendo um médico epidemiologista c um auxiliar de estatística, bem como representantes dos Consclhos de Medicina c Enfermagem, da Socicdade de Ginccologia e Obstetrícia do Paraná, profissionais da árca de epidemiologia e assistência matcrno-infantil do SUS, professores universitários da área de obstetrícia, para os quais se divulgou a proposta de formação dos comitês. Nasce aqui o caráter intcrinstitucional c multi-profissional que nortcia os comitĉs no Paraná.

O elemento chave dos comitĉs no Paraná tem sido o auxiliar de cstatística das Regionais de Saúde da Secretaria de Estado. Estc profissional recebe c controla todas as declarações de óbitos enviadas pelo cartórios e as repassa aos comitês. além de auxiliar na investigação dos óbitos ocorridos nos municípios mais distantes de sua regional. Os demais integrantes, médicos, enfermeiros, assistentes sociais, procedem à investigação $\mathrm{e}$ à análise dos referidos óbitos, além de emitirem parecer sobre a responsabilidade c evitabilidade das mortes matemas.

\section{Comitês de Morte Materna:}

São comitês de caráter interinstitucional, multiprofissional e confidencial que visam identificar dentre os óbitos de mulheres em idade fértil aqueles maternos c apontar medidas de caráter cducativo, normativo, administrativo, etc.

\section{Finalidade e função dos Comitês:}

- triar dentre os óbitos de mulheres cm idade fértil, os declaradamente maternos, presumíveis, procedendo à investigação;

- pesquisar a situação em que ocorreu o óbito, através de entrevista com o profissional que atendeu o caso e entrevista com a família:

- avaliar os aspectos de preventividade da morte, definir se o óbito era ou não cvitável:

- analisar se a responsabilidade pelo óbito foi: da pacientc, da assistência médica, da assistência hospitalar. social, inconclusiva, inevitável ou ignorada:

- propor medidas de prevenção de acordo com a análise da responsabilidade:

- corrigir cstatísticas oficiais e divulgar através de informações médicas e outros meios, como ofícios ou rclatórios. para todas as instituições e órgãos competentes que possam intervir na redução das mortes maternas;

- propor normas de funcionamento dos serviços, visando a melhoria da qualidade da assistência.

\section{Objetivo dos Comitês no Paraná:}

- identificação c quantificação das mortes materna;

- correção das estatísticas existentes, através da análise dos óbitos de mulheres em idade fértil;

- proposição de ações de saúde visando a redução dos cocficientes de mortalidade materna em $50 \%$ até o ano 2()()();

- scnsibili/ação dos órgãos oficiais e da comunidade sobre a magnitude do problema, objetivando sua participação na busca de doenças.

\section{Caráter dos Comitês:}

Fundamentalmentc, os comitês são interinstitucionais, multi-profissionais e devem atuar com características técnico-científicas, sigilosas, não coercitivas ou punitivas. A punição não é um mecanismo eficaz para a redução da mortalidade materna. Fica evidente que os comitês têm uma função essencialmente educativa, visando a prevenção dos condicionantes do obituário matcrno.

\section{Composição:}

Os Comitês do Paraná tem como ponto funndiamental a participação de pessoas de várias unsıluıçòes, que estejam relacionadas com o atendminento na área materna, ou que tenham grande capacidade técnica para desempenhar a referida função. A formação básica dos mesmos é a seguinte:

- médico ou enfermeiro epidemiologista - representando o SUS;

- representante do CRM (Gineco-Obstetra);

- representante do COREN (Enfermeiro);

- auxiliar de Estatística - SUS

- assistente Social - SUS

- representante dos movimentos organizados de mulheres.

A enfermagem está representada em todos os comitês, seja como representante do COREN, ou da equipe de Vigilância Epidemiológica da Secretaria Estadual, ou das Secretarias Municipais de Saúde. 


\section{APRESENTAÇÃO DOS RESULTADOS E ESTRATÉGIAS DE AÇÃO}

Os relatórios do Comitê Estadual de Morte Materna do Paraná, referentes aos anos de 1989 e 1990, foram elaborados a partir dos dados coletados através das "fichas de investigação confidencial de óbitos maternos". As mesmas são preenchidas pelos membros dos Comitês Regionais, que após a investigação de todos os óbitos de mulheres em idade fértil, através de pesquisas em prontuários hospitalares, de entrevistas com profissionais que atenderam a paciente ou de entrevistas com familiares nos domicílios, confirmam o óbito materno, ou descartam a presença de estado gestacional por ocasião do óbito. Nos casos confirmados preenche-se a ficha própria e posteriormente encaminha-se para o Comitê Estadual, que realiza um Seminário anual para apresentação da situação da mortalidade materna no Estado; os Comitês reúnem-se mais uma vez, para discutir e propor recomendações que visem a redução dessas mortes. (2)

Os dados referentes a 1991 estão em fase de processamento e foram analisados nesse ano 172 óbitos matemos.

Em 1989, no primeiro ano de implantação dos Comitês Regionais, apenas 16 Comitês apresentaram os resultados de suas investigações. Foram analisados nesse ano 90 óbitos matemos, sendo que destes, 51 $(56,7 \%)$ estavam declarados no atestado de óbito e 39 $(43,3 \%)$ óbitos foram descobertos pelos comitês. As causas obstétricas diretas foram identificadas em $71 \%$ dos óbitos (64 óbitos), as obstétricas indiretas em $18 \%$ e 10 óbitos eram não obstétricos. (Figura 1)

Os óbitos analisados corresponderam a $60 \%$ do total de óbitos maternos (139) ocorridos nesse ano.

Em 1990, com uma atuação mais efetiva dos Comitês, foram analisados 198 óbitos, sendo 177 obstétricos e 21 não obstétricos. Dos óbitos maternos obstétricos, 117 estavam declarados e $60(33,9 \%)$ não estavam declarados no atestado de óbito. (Figura 2)

As principais causas de óbito identificadas pelos comitês nesse período foram hipertensão específica da gravidez ( $50 \%$ dos casos), hemorragia, inf ecção e aborto. (Figuras 3 e 4 )

Nos dois anos estudados a maior freqüência dos óbitos foi na faixa etária de 25 a 35 anos, e apresentaram maior risco de óbito, as mulheres acima de 45 anos.

Em relação às condições sócio-econômicas das mulheres que foram a óbito, avaliadas em função da
Figura 1 - Óbitos maternos investigados: distribuição por causas obstétricas e não obstétricas, Paraná - 1989.

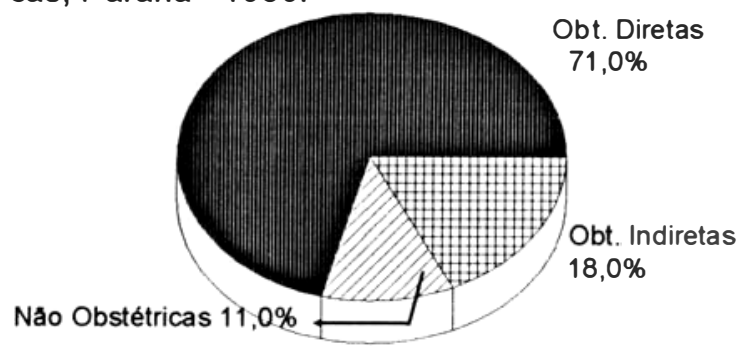

Comité Estadual de Morte Materna do Paraná, CISMC/SUS.

Figura 2 - Óbitos maternos investigados: distribuição por causas obstétricas e não obstétricas, Paraná - 1990.

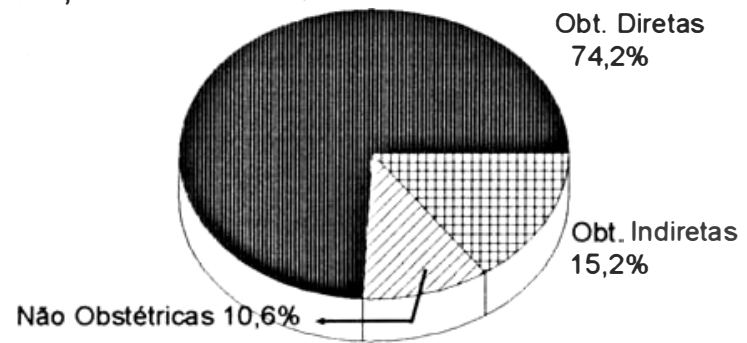

Comité Estadual de Morte Materna do Paraná, CISMC/SUS.

Figura 3 - Óbitos maternos obstétricos diretos segundo distribuição pelos grandes grupos de causa, Paraná - 1989.

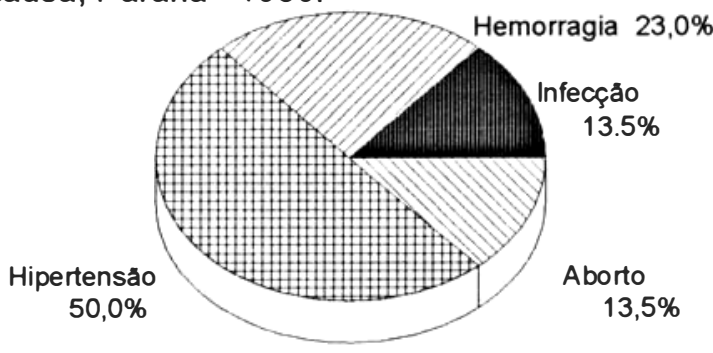

Comitê Estadual de Morte Materna do Paraná, CISMC/SUS

Figura 4 - Óbitos maternos obstétricos diretos segundo distribuição pelos grandes grupos de causa, Paraná - 1990.

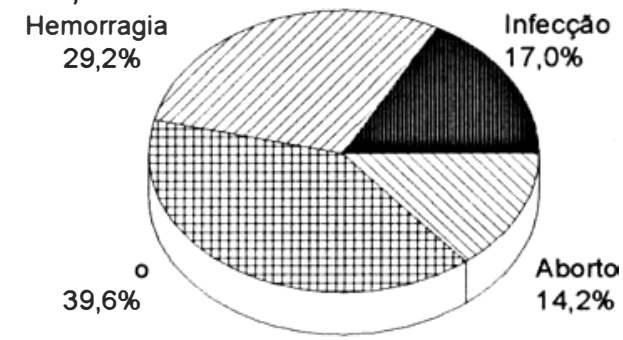

Comitê Estadual de Morte Matema do Paraná, CISMC/SUS. 
renda familiar e grau de instrução, verificou-se que cerca de $90 \%$ das mulheres tinham renda inferior a 3 salários mínimos. A média de percepção de renda familiar foi de 1 salário mínimo e a média de escolaridade foi de 3 a 4 anos, o que caracteriza os reflexos da influência dos fatores sócio-econômicos na determinação desta mortalidade.

Em 1989, 6\% das mulheres que foram a óbito tiveram parto domiciliar, e em 1990 apenas $4 \%$. Dos partos hospitalares em 1989, 53\% foram partos cesáreas, e em 1990 58\%. Nos casos em que foi possível identificar as condições do concepto através do prontuário ou de entrevista domiciliar, verificou-se que $20 \%$ dos resultou em nati ou neomorto.

Ao avaliar-se a evitabilidade dos óbitos maternos, considerando aquelas mortes evitáveis sob os conhecimentos atuais da medicina, o Comitê Estadual concluiu que em média, $89 \%$ dos óbitos eram evitáveis.

Conforme os critérios de classificação de responsabilidade pelos óbitos, da AMA - Associação Médica Americana, atribuiu-se ao médico e sua equipe $42 \%$ do obituário; $17 \%$ às medidas sociais; $16 \%$ às falhas administrativas hospitalares.

Estas análises permitiram caracterizar que as medidas preventivas necessárias, são de âmbito social e administrativo em $50 \%$ dos casos e outros $50 \%$ foram considerados preveníveis por medidas obstétricas.

Segundo a Organização Panamericana de Saúde, as estratégias destinadas a melhorar a saúde materna requerem uma ampla gama de ações que incluem: (5)

- atualizar a legislação referente às mulheres, incluindo as adolescentes, de forma que contemple seu direito à atenção e à proteção de sua saúde sexual e reprodutiva;

- estimular atividades comunitárias e dos grupos organizados de mulheres, a fim de propiciar o melhor conhecimento das ações mais eficazes para promover a saúde;

- desenvolver programas integrais de regulação da fecundidade, dirigidos de forma irrestrita tanto a homens como a mulheres, que incluam informação completa e atualizada sobre os métodos mais seguros e eficazes;

- capacitar o pessoal de saúde em relação aos aspectos de saúde da mulher e materna, incorporando estas atividades em todas as instituições de formação de pessoal de saúde.

Ainda segundo a OPAS, a mortalidade materna e sua redução é um dos grandes problemas por resolver na região das Américas, e para isto, é necessária uma clara vontade política e uma união de esforços e conhecimentos técnicos de líderes, tanto do setor saúde como de outros setores, para chegar a soluções efetivas. (5)

Dentro das medidas que são necessárias para alcançar este objetivo figuram: melhoria dos serviços de saúde; educação da comunidade; maior integração dos serviços de planejamento familiar nos programas de saúde materna; melhor cuidado ao parto e ao período pré e pós neonatal; tratamento adequado das complicações da gravidez e do parto. A organização dos serviços de saúde deve ser enfocada de acordo com distintos níveis de atenção, com o objetivo de potencializar sua eficiência e acessibilidade, diminuindo os custos.

A implantação dos Comitês de Morte Materna, vem sendo insistentemente proposta pela OPAS, como uma das estratégias para a redução da mortalidade materna, pois estes visam analisar periodicamente as informações sobre a situação das mortes matemas, para facilitar a proposta de medidas imediatas. ${ }^{(6)}$

Quando os objetivos acima forem alcançados, se cumprirá uma das metas da Organização Panamericana de Saúde e expressa nas "estratégias regionais para o futuro" sobre a saúde da mulher e o desenvolvimento: "Reduzir as inaceitáveis taxas de mortalidade materna nos países em desenvolvimento." Desta maneira, não só se beneficiarão as mães e seus filhos, como também se melhorará a saúde e se incrementará o bem estar de toda a população."

\section{CONCLUSÕES}

O enfermeiro tem se mostrado um profissional necessário e competente como membro dos Comitês de Morte Materna do Paraná, por estar envolvido com as ações de saúde destinadas à mulher, desde os níveis de execução ambulatorial ou hospitalar, passando pela formação de recursos humanos nas escolas de enfermagem de nível superior ou médio, bem como participando de equipes multiprofissionais nas áreas de plane jamento e avaliação dos programas de saúde materna.

Os Comitês de Morte Materna abrem um novo espaço de trabalho aos enfermeiros, que é a pesquisa epidemiológica de outros agravos à saúde, além das doenças transmissíveis de notificação obrigatória.

A vigilância epidemiológica e/ou auditoria das 
mortes matemas. realizadas pelos comitês, permite o estudo constante do problema, a análise dos dados estatísticos gerados, a identificação das causas mais comuns, e permite pela sua sistematização, a geração de um conhecimento sobre a realidade, além da sensibilização de profissionais diretamente envolvidos no processo de assistência à mulher, bem como das autoridades sanitárias e da comunidade.
No Paraná, a enfermagem está representada $\mathrm{cm}$ todos os 24 Comitês Regionais e no Estadual, realizando esta importante tarefa, junto aos demais membros dos Comitês, de estudar e analisar os óbitos maternos, e propor medidas de ação para a redução da mortalidade materna em suas áreas de atuação.

\section{REFERÊNCIAS BIBLIOGRÁFICAS}

1. COMITÊ ESTADUAL DE MORTE MATERNA. Manual dos Comitês de Morte Materna. Curitiba, junho, 1992. (Versão Preliminar).

2. COMITE ESTADUAL DE MORTE MATERNA. Relatório Bi-Anual- 1989 e 1990 . Curitiba.

3. LAURENTI, R. A Mortalidade Materna - Estado de São Paulo: sua evolução nas últimas três décadas. Trabalho apresentado no Simpósio Franco-Brasileiro sobre prevenção da mortalidade materna. Junho, 1992, (mimeo).

4. OMS. Mortalidade Materna: Ayudar a las mujeres a evitar el camino de la morte. Separato de crônica de $1^{\mathrm{a}} \mathrm{OMS} 40^{(5)}$ : 195-205, 1986.
5. ()PAS. La Salud Materna: um perene desafio. Sociedade publi saciones: Comunicación para la salud. $n^{\circ} 1,1991$.

6. OPAS/()MS. Estratégias para la prevencion de la mortalidad mate na em las Américas. Agosto, 1989.

7. (OPS ()MS. Plan de accion regional para la reduccion de la murtalidade materna en las Américas. Setembro, 1990.

8. TANAKA, A C. d'. Importancia de um sistema de vigilancia epidemiologica na mortalidade materna. Trabalho apresentado na $2^{a}$ Reunião dos Comitês de Morte Materna do Puraná. Agosto, 1991. (mimeo). 